\title{
The relationship between Righteousness and Disease
}

\author{
Jianzhong Sun ${ }^{1,}$, Jiaojiao jia ${ }^{2, \text { b, }}$, Mingsan miao ${ }^{3, c, ~ *}$ \\ ${ }^{1}$ Department of pharmacology of Chinese materia, Henan University of Traditional Chinese \\ Medicine,Zhengzhou, 450046, China \\ ${ }^{2}$ Department of pharmacology of Chinese materia, Henan University of Traditional Chinese \\ Medicine,Zhengzhou, 450046, China \\ aemail: sunjianzhong@163.com, bemail:594872967@qq.com, \\ Cemail:miaomingsan@126.com, corresponding author
}

Keywords: Righteousness, Disease ,Immune

\begin{abstract}
Describes the meaning of righteousness, explores and research the relationship between righteousness, disease and human body,a new viewpoint is put forward for the future health concept and the application of medical assistant means. The medical treatment, aftercare, exercise are the means assisted to improve the righteousness, the main factor that the body to maintain the health is the righteousness of human body itself, under the joint action of other auxiliary means, to ensure the human health.Righteousness is the human body to maintain in the internal state of health, exercise, health and medicine is external auxiliary, the correct concept of health is the guarantee to ensure our health.
\end{abstract}

\section{Introduction}

From the point of view of the traditional Chinese medicine, we should understand,disease is a special stage of human body, excessive medical treatment will not bring good results, but will have excessive dependence, so that the body's own defense has no chance to application. Treatment of the disease should be based on "people", "people" is the protagonist, transfar and outcome of disease are dominated by people, and varies from person to person.righteousness is the dominant factor in the healthy development of the body, is the main contradiction. Other means are auxiliary factors, is the secondary contradiction. We should grasp the main contradiction, and carry out further in-depth discussion on its development.

\section{Righteousness}

Body of the gas, is a human body of the various organs, and running on the whole body of very subtle substances[1].From the essence of nature, food and the nature gas three fusion into,with defense, anti pathogen, regulation, rehabilitation and other functions. From the view point of modern study righteousness is the normal function of the human body and produced a variety of maintenance ability of health, including self adjustment ability, ability to adapt to the environment, anti evil disease prevention and rehabilitation self-healing ability and so on, the approximate to we often say that the body resistance and immunity [2].

The same environment, righteousness deficiency are more likely to feel the pathogenic factors and pathogenesis,righteousness exuberant person is not easy to invaded, the human body is also won't get sick, here is the representation of the differences of righteousness .Some scholars believe that righteousness is a kind of any physiology and structure in a normal range to maintain the 
functional material, close to the western medicine "dynamic balance of reserve". Functional maintenance or restoration of different levels of physiological - psychological - immune - secretion - social - mental health state (subcellular, multicellular, tissue, organ, system, physical, social and mental), so that the human body from the "sub health" to "healthy" state.And the healthy function and strength is the individual, and it changes from age[3].Some people think righteousness is the unity of human tissue structure and physiological function of, refers to the human body to resist pathogenic factors and maintain the organizational structure and physiological function of normal life activities of the organism as a whole.

"Evil influence" is an important concept in the theory of traditional Chinese medicine. Generalized evil influence is the floorboard of a variety of pathogenic factors; the narrow sense of evil influence refers to some specific virulence factors[4]. Only when the body righteousness relatively weak not enough to resist the evil influence, it can take advantage of a weak point, violations of the human body, cause viscera imbalance of yin and Yang, qi movement disorders and disease.

The occurrence of diseases related to the two aspects of righteousness and evil influence, The occurrence and change of disease is the reaction of the struggle between righteousness and evil influence under certain conditions.

\section{Disease initiation, initial development, prognosis and recovery}

The thought of "treatment of the disease has not yet occurred" emphasis on disease prevention first, to improve the righteousness of body, enhance the body resistance to disease.Righteousness can resist disease pathogen invasion, to get rid of evils and prevent disease,specific performance in: to resist external evil invasion, eliminate disease, repair adjustment ability, to maintain the coordination of internal organs and meridians.Chinese traditional Chinese medicine believes that the imbalance of yin and Yang, and the righteousness growth and decline are the basic mechanism of the disease. The cause of the disease is the cause of the disease, including the pathogenic factors, pathogenic characteristics, disease characteristics, etc..Righteousness will anti pathogenic when the pathogen invasion. If the righteousness is strong enough to beat the evil, the body will not show clinical symptoms and signs,otherwise exogenous diseases take advantage of the weak point so that disease occurrence.The intrinsic factors of disease initiation is thus lack of righteousness.

The occurrence and change of the disease is complex, in general,Is a struggle between the righteousness and the evil influence.Righteousness is the decisive factor in the course of the struggle,which is one of the characteristics of traditional Chinese medicine[6].Evil invasion,righteousness is not strong enough to resist the evil,caused Yin and Yang imbalance, dysfunction. The body has a disease, clinical symptoms and sign.If righteousness is strong enough to have a fierce struggle with the evil influence,the body will show up with sthenia syndrome.If the righteousness is weak,can not beat the evil,the evil influence will come into the organs deeply,the body will show up with intensive syndrome mostly. The thought of "treatment of the disease has not yet occurred" emphasis on that early diagnosis and early treatment should be done to prevent the development of the disease at the initial stage of the disease.

In the process of development and change of disease, the righteousness and the evil influence two forces are constantly changing. There are changes of the ups and downs of the struggle process, this change plays a decisive role on the prognosis of the disease. Righteousness growth and exuberant, evil influence subsided and attenuation, disease trends to turn for the better, otherwise disease trends to deteriorate, even lead to death. According to the thought of "treatment of the disease has not yet occurred",In the early stage of disease, the disease is relatively shallow, light 
condition, righteousness is not weak, so that the disease is easy to cure, and thus less transmission. In the mastery of disease occurrence law of development and transmission way based on, early diagnosis and early treatment to prevent disease development, blocking the spread of the disease pathway.Body encountered pathogen attack, righteousness rise against and defeat it,disease recovery.Evil hyperactivity and righteousness weak, body unable to resist pathogens, disease gradually tended to deteriorate in critically ill, and even lead to the outcome of death,evil wins.Evil is not excessiveness, righteousness is not weak, each sticks to its own stand, righteousness cannot eliminate evil completely, evil stay in a part of the body, eventually caused chronic disease or syndrome sequelae.or strengthening the body resistance to eliminate pathogenic factors, illness can be improved and cured.

The thought of "treatment of the disease has not yet occurred", Not only emphasized "often known as variable, know about the changes to prevent" the scientific philosophy of dialectical materialism, the more prominent the thoughts of rehabilitation medicine "not only lead to disease, but also recurrence after cure". The deficiency of righteousness is the internal factors of the disease,therefore the treatment for the disease has not yet occurred,must be started from strengthening the human body righteousness.Cause righteousness itself has the function of driving the evil influence out and rehabilitation.If the evil influence is weaker than righteousness,some illness can be healed even without the use of drugs.Hold up the righteousness means to strengthen the physique, improve the body's resistance to evil and the ability to recover. Applicable to all kinds of deficiency syndromes, helps the body to get rid of the disease. When the disease after initial healing,righteousness has not been fully restored, at this time, to take for recuperation method patients, promote the rehabilitation of the body.

Rehabilitation is the physiological function to improve or recover the body's organs of the body, that is to use all kinds of measures to solve the various factors caused by congenital or acquired organs function decline or dysfunction of medical treatment, so as to improve their physiological function or recovery.Chinese medicine emphasizes the holistic rehabilitation principles. advocates to consider people in the overall environment, make full use of concatenates, mutual induction, pull the whole relationship between the overall situation, Recovery through the whole essence of Yin and Yang and blood conditioning viscera physiological function, on the basis of syndrome differentiation and treatment,to achieve the purpose of rehabilitation.It grasps the overall functional status and functional structure of the human body, in essence, is to help people disease resistance.Mobilize the active rehabilitation ability, such as the use of food therapy, acupuncture and other methods to cure illness, through adaptation to seasonal climate changes, change eating habits to promote somatic disease rehabilitation.

Sub health status is a disease state between disease and health, although there are various symptoms, but can not reach the diagnostic criteria of disease, this state is the body in a low quality healthy state.It has two trends: if intervene in the sub healthy state reasonable, can make the body to restore the health status; conversely, if the body is in a long-term sub-health state, it may lead to a series of diseases.The thought of "treatment of the disease has not yet occurred" of TCM emphasizing the people as a whole, emphasizing the unity of man and heaven and earth.You should conform to the natural gas if you want to restore the human body righteousness, Emphasize the similarities of human and society groups and the integration of human and the nature. Through the body health, to achieve health and longevity and disease prevention. 


\section{Righteousness and Disease}

Disease can be divided into three cases: 1 . it will be cured with or without a doctor; 2 .it will not be cured with or without a doctor ; 3 .it will be cured with a doctor. The first category is "false sick",also named "disease has not yet occurred". This kind of patient to go to a doctor, the doctor must give you some medicine, all the drugs have side effects, will be more or less weaken the body's self-healing function, but also may bring "medical source disease", so some people say that this is "a hundred harm and no benefit". So some people say that this is "a hundred harm and no benefit". Only the second and the third categories are real diseases. These two kinds of categories only accounted for $10 \% .90 \%$ of the disease belongs to the "false sick", it will be cured with or without a doctor.

Human body has a cure the disease ability through internal adjustment to balance and self repair.Traditional Chinese medicine often said, "disease by drug treatment accounted for $30 \%$, by self cultivation accounted for 70\%" also shows that traditional Chinese medicine is not too dependent on drugs, but focusing on the mobilization of the body's self-healing power. during the crashes in the dysfunction of organs, instability of internal and external environment, the human endogenous regulation system will play the role of self-regulation, and produce, release more righteousness, to repair, alteration, eliminate hidden dangers and the lesions of the body. There are more cases of self healing in clinical cases reported, such as psoriasis, erythema, and even can not be cured of cancer [8].Righteousness has been used as an important index to judge the disease healing,our ancient ancestors attaches great importance to improving healthy by means of acupuncture, to achieve the purpose of preventing and curing diseases[9].

Seeking the nature of medical treatment is the basic principle of syndrome differentiation and treatment.Positive treatment and anti treatment are two kinds of treatment under the guidance of the basic principle of "seeking the nature of medical treatment",based on the disease nature and the phenomenon .Surface and essence,primary and secondary are different in the development process of the disease. The treatment principle should be applied flexibly according to the actual situation.

The outcome of the struggle between righteousness and evil influence, determine the disease development and prognosis, if righteousness won the fight, evil influence would be gone, otherwise, the illness would damage the body.Hold up the righteousness and eliminate the evil influence is the important rule of clinical treatment guidance, strengthening the body resistance to eliminate pathogenic factors in the course of treatment, and assist the body righteousness to cure the disease, and righting the body healthy energy increases and helps in the body to fight off evil, conducive to the preservation of righteousness and recovery[10-12] .

The human body is a whole system, the righteousness strength and self repair capacity determines the outcome and prognosis of illness.In the treatment of the disease, why in clinical regardless of how clever medicine doctor, treatment of disease some can be cured, but some cannot be cured even invalid? This shows that in addition to the doctor's diagnosis and treatment is appropriate, but it is mainly depends on the patient's own immune function status and repair capacity [13]. All medical and drug is only a means to treat the disease, the real objective factor is determined by the human body itself.

The immune system is a function of the body to recognize and eliminate foreign antigens and maintain its physiological balance and stability, which is the three major function of defense, self stabilization and surveillance.in simple terms, the concept of immunity means exclusion of "non self", save "itself",and the motherland medicine "righteousness" effects are almost the same, and human lung, spleen and kidney are closely related[14]. Lung mainly related to fur, plays a role in 
the barrier, is the first line of defense against the invasion of evil.In the immune system, the spleen belongs to the peripheral immune organs, and it is the location of the lymphocytes. The bone marrow is the central immune system in the immune system, which is the origin of the lymphocytes. The source of kidney and immune active cells is directly related[15].Immunity can be in connection with the theory of "righteousness and evil influence", normal immune system function is stable,keep the body from the pathogen, belong to the category of "righteousness";Excessive activation of inflammatory cytokines and inflammatory medium should belong to the category of "evil influence"; reduce the immune substances, immune imbalance and immune dysfunction will cause the pathological changes of the body.People will get sick if the human body immune imbalance, the body against the pathogen's ability to drop, righteousness weak .

The body of "righteousness" and "immune" both can resist pathogen,Its rich or not directly affects the resistance ability and the level of health of body.Modern medicine is to improve people's immunity, can carry out targeted vaccination, so as to improve the specific or non-specific immune function [17]. "Congenital" is an important aspect of righteousness filling. In addition, acquired nursed back to health, exercise, appropriate ancillary drugs can also be used to support healthy, enhance the function of the immune system. But these means are auxiliary functions, and can not replace leading role of righteousness in the course of human health development and against the disease, grabbed the internal factor, solving the main contradiction, any problems will be solved.

However righteousness is not specific of a material composition, is not an organ with the function, it is a holistic concept, is the unity of substance and function. At present, many scholars have related the righteousness to modern immunology, righteousness has the role of modern immunology.This definition from the function of righteousness has certain significance for Understanding of righteousness, but there is a big problem to explore the core content of righteousness. Therefore, from the holistic view of TCM,righteousness of material properties and the physiological and pathological characteristics, may promote our understanding of righteousness.

From the holistic concept of traditional Chinese medicine,righteousness includes both constitute the human body and maintain the life activities of the human body nutrient substances.It also includes the maintenance of physiological functions of the body's normal life activities.It is the unity of matter and function, and it is the general name of the function of life. Righteousness resists exopathogens, maintains normal life activities before disease came up.After disease, it is responsible for the task of eliminating the evil influence, strengthening the body healthy.From a functional perspective, in traditional Chinese medicine, righteousness is not only include the concept of modern immunology, the more important it is also the nutrient substances that constitutes the human body and maintains the life activities.Righteousness is the organic integration of the nutrient substance, but it is not a simple sum and cannot exist independently. It is through each part of the interdependence, mutual to grow jointly maintain the body's normal life activities of the whole matter。

\section{Conclusion}

A variety of reasons make people get sick, but in the view of TCM,a person is healthy or not, depends on the righteousness and the evil influence. Cultivation of righteousness process, can be said is the process of nourishing of life.It is a process of abstemious daily life, peace of mind, proper exercise, to keep the body healthy and balanced development.The role of auxiliary means is not the direct effects on the human body, but that regulates the body's imbalance, auxiliary righteousness to mobilize the body to resist evil ability, namely the healing power, so as to eliminate the disease.In the cause of the rapid development of science and technology of the moment, we 
should use auxiliary means to fully mobilize the body's healing power, enhance its ability to protect,treat the body as the protagonist, figured out the main contradiction and secondary contradiction, clear internal and external. Make full use of the self-healing ability of the body to the prevention and treatment of diseases, and not excessive dependence on even in order to the auxiliary means as the center, put the cart before the horse.

\section{Acknowledgement}

In this paper, the research was sponsored by the Henan province university science and technology innovation team (2012IRTSTHN011); Zhengzhou science and technology innovation team (131PCXTD612).

\section{Conference}

[1] $\mathrm{Xu}$ Ning.the meaning of "righteousness" in $<\mathrm{Nei}$ Jing $>$. [J].Shaanxi traditional Chinese Medicine,2009,30(9):1186-1188.

[2]Wang Cihua.The traditional "evil influence" is the pathogenic factors. [J].Public health journal,2013-11-14(010).

[3] Roberto Gonzalez,Niu Xin. Based on the basis of modern medicine righteousness interpretation . [J].The Chinese medicine medicine magazine ,2012,27(6):1541-1543.

[4]Liu Lu, Zhu Wanping, Kong Fanzhi, et al. The discuss of "evil influence" in <Ling Shu>. [J].Journal of Liaoning University of Traditional Chinese Medicine,2014,16(11):77-80.

[6]Jiang Yong,Chen Jianshan, Jiang Ruiyun, et al.On the complete system of syndrome differentiation and treatment. [J]. people - syndrome and disease.Journal of Traditional Chinese Medicine,2011,52(17):1447-1450.

[8]Wang Qiangfen, Zou Mingliang. Study on the research of the human body's self-healing ability in Chinese and western traditional medicine. [J]. Chinese Journal of traditional Chinese medicine, 2011,17(2):213-214.

[9]Dai Fei, Wang Chao, Guo Yi. Discussion on the excitation of the human body to stimulate the body's self healing power. [J]. Sichuan traditional Chinese medicine,2014,32(2):37-38.

[10]Zheng Zuohuan, Ju Baozhao. "HuangDiNeiJing" principle of treatment research. [J]. Journal Liaoning University of Traditional Chinese Medicine,2014,16(1):88-91.

[11]Liu Xiangbin. The prevention and cure effect of on the sub health of the evil . [J]. Journal of traditional Chinese medicine,2014,26(9):955-956.

[12]Xiao Kai, Miao Mingsan. Traditional Chinese medicine research of Qi. [J]. Journal of Chinese medicine,2014,29(192):718-720.

[13]Wei Huifang, Zhang Lufen. From the whole idea about the characteristics of traditional Chinese medicine of "righteousness" . [J]. Journal of Beijing University of traditional Chinese medicine,2009,32(7):440-442.

[14]Liu Weiqing. Relationship between immune and traditional Chinese medicine . [J].Shaanxi traditional Chinese medicine ,2003,24(7):629-632.

[15]Li Li Ping, Zhao Yagang.Study on the current situation of righteousness and the immunity, the micro ecological balance. $[\mathrm{J}]$. Journal of Chinese and western modern medicine,,2012,21(31):3524-3526.

[17]Zhang Xuefeng. Chinese "righteousness" and "immune" in modern association. [J]. Jiangsu 
health care,2004,6(3):47-48. 